Check for updates

Cite this: RSC Adv., 2018, 8, 24084

\section{The anticancer efficacy of paclitaxel liposomes modified with low-toxicity hydrophobic cell- penetrating peptides in breast cancer: an in vitro and in vivo evaluation}

\author{
Qi Zhang, ${ }^{a}$ Jing Wang, ${ }^{a}$ Hao Zhang, ${ }^{a}$ Dan Liu, ${ }^{a}$ Linlin Ming, ${ }^{b}$ Lei Liu, ${ }^{a}$ Yan Dong, ${ }^{c}$ \\ Baiyu Jian ${ }^{\mathrm{c}}$ and Defu Cai (D) *a
}

In our recent study, hydrophobic cell-penetrating peptides (CPPs) were demonstrated as an effective method of improving cancer treatment. To provide more evidence and broaden the application range for this promising strategy of improving cancer treatment, novel hydrophobic CPP-modified (PFV-modified) nanoliposomes loaded with paclitaxel, termed PFV-Lip-PTX, were developed as a treatment for breast cancer. Physicochemical evaluations of PFV-Lip-PTX revealed spheroid-like regular vesicles of about $120 \mathrm{~nm}$ in diameter with negative charge. An in vitro release study indicated that PTX was released from the liposomes in a controlled and sustained manner. A cellular uptake study indicated that PFV-Lip-PTX exhibited higher internalization efficiency in MCF-7 cells than non-modified liposomes. It was also demonstrated that PFV modification improved the cytotoxicity of PTX via a hydrophobic interaction between the PFV-Lip and cell lipid membranes compared with non-modified liposomes. Moreover, in vivo studies demonstrated that the PFV-modified liposomes led to highly efficient targeting and accumulation in an MCF-7 xenograft tumor and improved the antitumor efficacy of PTX. Finally, PFV-Lip-PTX showed low systemic toxicity evidenced by fewer changes in the body weights of mice and no visible histological changes in major healthy organs. Therefore, our results indicate that PFV-Lip-PTX has great potential in tumor-targeting and effective antitumor treatment.
Received 26th April 2018 Accepted 18th June 2018 DOI: $10.1039 / c 8 r a 03607 a$ rsc.li/rsc-advances breast cancer, non-small cell lung cancer, stomach cancer, head and neck carcinomas, advanced forms of Kaposi's sarcoma and acute leukemia. ${ }^{4,5}$ PTX exerts its action through binding to the $\beta$-subunit of tubulin, a component of microtubules that results in the formation of stable microtubule assemblies. ${ }^{6}$ Subsequent PTX blocks the mitotic checkpoint and arrests cells in the late G2 and M phases of the cell cycle. ${ }^{7}$ However, the significant activity of PTX in clinical applications is greatly restricted by its poor water solubility, low therapeutic index and severe side effects at high doses. ${ }^{8}$ Due to the low water solubility of PTX in conventional formulations, a mixture of Cremophor EL and dehydrated ethanol $(50: 50, \mathrm{v} / \mathrm{v})$ was applied as a co-solvent in the first clinical formulation of PTX under the trade name of Taxol®. ${ }^{9}$ It is characterized by very low drug loading ( $1 \mathrm{wt} \%$ ). Therefore, substantial amounts of Cremophor EL and ethanol are necessary to ensure an effective dose of PTX. However, many serious side effects including neutropenia, hypersensitivity, anaphylactic reactions, nephrotoxicity and neurotoxicity have been attributed to Cremophor EL, which remarkably impede dosing and often require clinical intervention. ${ }^{\mathbf{1 0 , 1 1}}$ These negative side effects mean that the medical benefits obtained from PTX seem to have reached a plateau. Hence, the development of an improved delivery system for PTX is extremely desirable.
${ }^{a}$ Institute of Medicine and Drug Research, Qiqihar Medical University, Qiqihar 161006, P. R. China.E-mail: cai@qmu.edu.cn; Tel: +86-452-2663376

${ }^{b}$ The Third Affiliated Hospital, Qiqihar Medical University, Qiqihar 161006, P. R. China

${ }^{c}$ College of Pharmacy, Qiqihar Medical University, Qiqihar, 161006, P. R. China 
Fortunately, the introduction of nanotechnology in oncology could alleviate potential systemic toxicity and improve the therapeutic effect of PTX. A few successful nano-delivery formulations have already been accepted as alternative formulations of PTX on the market, such as Abraxane® (albuminbound PTX nanoparticles), Lipusu® (PTX liposome injection) and Genexol-PM® (PTX polylactide-polyethylene glycol micelles). ${ }^{12}$ Well-designed nanocarriers for the delivery of PTX to tumor tissues might possess numerous appealing properties, such as increased PTX solubility, the ability to promote accumulation in tumor sites via passive and/or active targeting, reduced side effects and controlled release. ${ }^{13}$ Among the various existing delivery systems, liposomes are the most promising for clinical applications, as they exhibit high delivery efficiency, good biocompatibility, non-immunogenicity and the ability to encapsulate hydrophobic and hydrophilic drugs. ${ }^{\mathbf{1 4}}$ However, compared to Taxol, PTX liposomes do not show a significant difference in response rate, and can also lead to the development of hematological and nervous system toxicity in patients. ${ }^{15}$ Its clinical effect in increasing survival is still unclear. Usually, it is difficult to achieve good outcomes when using liposomes as a clinical treatment, as they often cannot efficiently enter tumor cells. ${ }^{16}$ To overcome this difficulty, some specific functional moieties have been attached on the surfaces of liposomes for further functionalization on the basis of passive targeting. ${ }^{17}$ Among the many proposed strategies for improved delivery of liposomes, cell penetrating peptides (CPPs) have appeared as an achievable strategy and have led to encouraging results. One of the most remarkable properties of CPPs is their capability to deliver bioactive cargo with molecular weights several times greater than their own through the cell membrane. ${ }^{18}$ Since the discovery of the first CPPs in 1988, a variety of CPPs derived from natural, chimeric and synthetic sources have been used to transport small RNA/DNA, plasmids, antibodies and nanoparticles into specific sites of different cells. ${ }^{19}$ However, most CPPs are positively charged and are classified as cationic CPPs, which present toxicity alongside cellular uptake improvement.

Hydrophobic CPPs have a low net charge, contain only nonpolar residues and have a hydrophobic motif that is crucial for uptake. They exhibit low toxicity to normal cells, which contrasts what is observed for most cationic CPPs. ${ }^{20,21}$ Moreover, some hydrophobic CPPs can directly translocate across lipid cell membranes, which could be especially advantageous as they would immediately get to the cytosol, eliminating the risk of endosomal entrapment. ${ }^{22}$ Several hydrophobic CPPs have been confirmed to be efficient in delivering therapeutic molecules into cells, such as the signal sequence from integrin $\beta 3$ (VTVLALGALAGVGVG), ${ }^{23}$ FGF (PIEVCMYREP) ${ }^{24}$ and a short sequence QLPVM. ${ }^{20}$ In our previous study, the typical hydrophobic CPP, PFVYLI (PFV), which is derived from $\alpha 1$-antitrypsin, was introduced to modify doxorubicin-loaded liposomes for the first time. The modified liposomes were capable of facilitating intracellular delivery as well as improving antitumor efficiency without apparent toxicity. ${ }^{25}$ Furthermore, PFV serving as a carrier could efficiently facilitate the intracellular transport of cargoes such as siRNA, fluorescent probes and proapoptotic peptides into a number of different cell lines. ${ }^{26-28}$ However, further proof is needed to support the conclusion that the introduction of hydrophobic CPPs is an appropriate choice to improve the intracellular delivery of nanocarriers.

To verify that functionalizing liposomes with PFV would facilitate their intracellular delivery via the hydrophobic interaction between PFV and cell lipid membranes, we synthesized a novel PFV-conjugated PEG-DSPE polymer and incorporated it into the liposomes. The prepared PFV-Lip was characterized in terms of particle size, zeta potential, morphology, encapsulation efficiency and drug release. The in vitro cellular uptake of the PFV-modified liposomes was investigated via flow cytometry, confocal microscopy and HPLC analysis on MCF-7 cells, and the in vitro cytotoxicity was determined through an SRB assay. Finally, the in vivo distribution and anti-tumor efficacy of PFV-Lip-PTX were investigated in MCF-7 tumor-bearing mice. The systemic toxicity of PFV-Lip-PTX after administration was also evaluated (Fig. 1).

\section{Experimental}

\subsection{Materials}

Paclitaxel (PTX) was provided by Dalian Meilun Biotech Co., Ltd. (Dalian, China). Egg yolk phosphatidylcholine (EPC, LIPOID E-

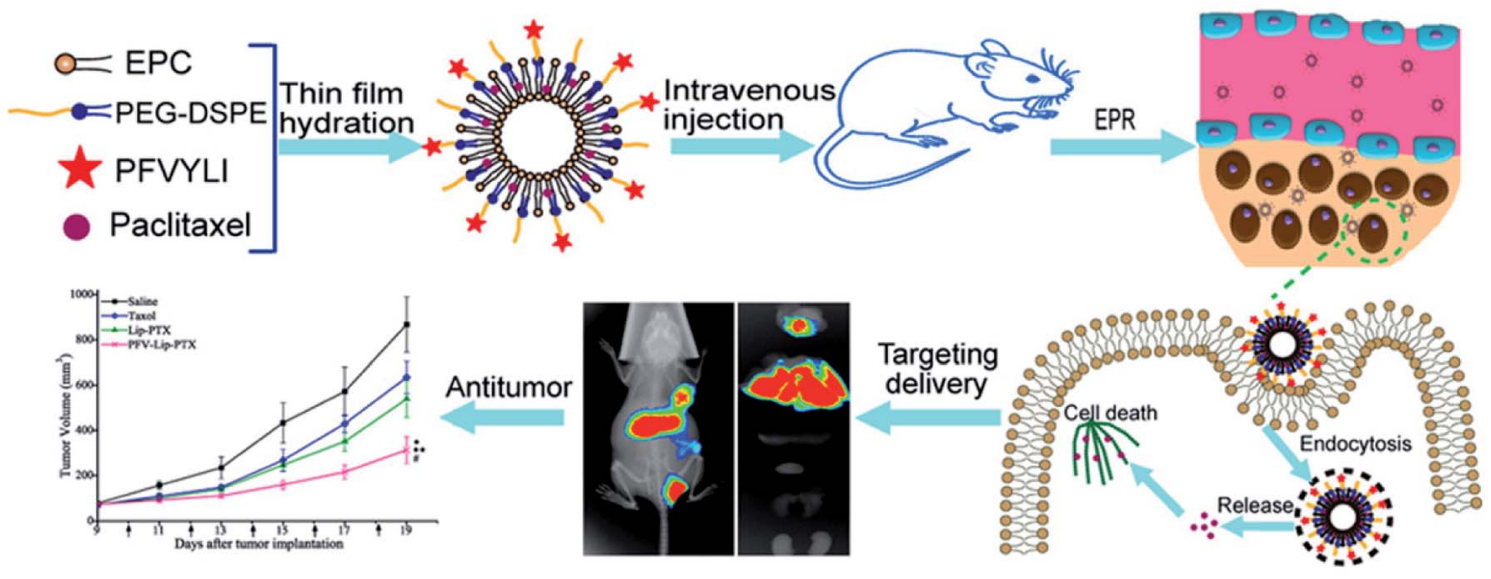

Fig. 1 Scheme of PFVYLI-modified liposomes for targeted delivery of PTX in vivo. 
80) was obtained from LIPOID GmbH (Ludwigshafen, Germany). Cholesterol (Chol), sephadex G-50, Cou6, Hoechst 33258, sulforhodamine B (SRB), trichloroacetic acid (TCA) and Tris base were all purchased from Sigma-Aldrich (Shanghai, China). Near-infrared lipophilic carbocyanine dye DiR was obtained from Biotium, Inc. (Hayward, USA). PEG $_{2000}$-DSPE and $N$ hydroxysuccinimidyl-PEG ${ }_{2000}$-DSPE were purchased from ToYong Biotech Co., Ltd. (Shanghai, China). PFVYLI was provided by China Peptides Co., Ltd. (Shanghai, China). All other chemicals and reagents were purchased from SigmaAldrich (Shanghai, China) unless otherwise stated.

Female BALB/c nude mice of 18-20 g were obtained from Beijing Vital River Laboratory Animal Technology Co., Ltd. (Beijing, China). Before the experiment, they were kept under SPF conditions for 1 week and were allowed free access to standard food and water. All animal procedures were performed in accordance with the Guidelines for Care and Use of Laboratory Animals of Qiqihar Medical University and experiments were approved by the Animal Ethics Committee of Qiqihar Medical University.

\subsection{Cell culture}

The human breast cancer cell line MCF-7 was obtained from the Institute of Basic Medical Science, Chinese Academy of Medical Science (Beijing, China). The MCF-7 cells were cultured in RPMI-1640 medium (Macgene, China) supplemented with 10\% FBS (Gibco, USA) and 1\% penicillin-streptomycin solution (Macgene, China) at $37{ }^{\circ} \mathrm{C}$ in $5 \% \mathrm{CO}_{2}$ air.

\subsection{Conjugation of PFV peptide with NHS-PEG-DSPE}

PFV-PEG-DSPE was synthesized in our laboratory according to a previously reported method. ${ }^{25} \mathrm{PFV}$ was conjugated with NHSPEG-DSPE through a nucleophilic substitution reaction. Briefly, PFV peptide and NHS-PEG 2000 -DSPE in a $1: 2$ molar ratio were dissolved in anhydrous DMF, and the $\mathrm{pH}$ of the reaction mixture was adjusted to 8.0-9.0 with triethylamine. The reaction was kept at room temperature under moderate stirring and the reaction was monitored by HPLC (Waters, USA). After $144 \mathrm{~h}$, the reaction product was subjected to dialysis (MWCO $3500 \mathrm{Da}$ ) against deionized water to remove the unconjugated PFV peptide. Finally, the dialysate was lyophilized and stored at $-20{ }^{\circ} \mathrm{C}$ until required. The product was identified using a MALDI-TOF mass spectrometry (Bruker Daltonics, USA).

\subsection{Preparation and characterization of various drug-loaded liposomes}

The thin-film hydration method was used to prepare the liposomes loaded with PTX or fluorescent dye (Cou6 or DiR) as described previously. ${ }^{29}$ Typically, $15.9 \mathrm{mg}$ of EPC, $4.1 \mathrm{mg}$ of Chol, $4.7 \mathrm{mg}$ of $\mathrm{PEG}_{2000}$-DSPE and $1.5 \mathrm{mg}$ of PFV-PEG ${ }_{2000}-\mathrm{DSPE}$ were used in the preparation of the PFV-modified liposomes. For the unmodified liposomes, $15.9 \mathrm{mg}$ of EPC, $4.1 \mathrm{mg}$ of Chol, and $6.0 \mathrm{mg}$ of PEG $_{2000}-\mathrm{DSPE}$ were used. $1.0 \mathrm{mg}$ of PTX or $10 \mu \mathrm{g}$ of fluorescent dye were added into the liposomal compositions to prepare drug-loaded or fluorescence-labeled liposomes, respectively. Lipid mixtures with PTX or fluorescent dye were codissolved in $35 \mathrm{~mL}$ dichloromethane, and the organic solvent was removed by rotary evaporation. Then the obtained film was further dried and stored under vacuum overnight. The lipid film was hydrated with phosphate buffer $(\mathrm{pH}$ 7.4), followed by sonication at $37^{\circ} \mathrm{C}$ for $1 \mathrm{~h}$ until a clear solution was obtained. The liposome suspension was extruded through polycarbonate membranes with a pore size of $220 \mathrm{~nm}$ followed by gel filtration over a Sephadex G-50 column to remove the unencapsulated PTX or fluorescent dye. The liposomes were stored at $4{ }^{\circ} \mathrm{C}$ and used within 2 weeks of preparation.

The particle sizes and zeta-potentials of different liposomes were determined by dynamic light scattering (DLS) using a Nicomp 380ZLS (PSS, USA). The analysis was performed with $633 \mathrm{~nm}$ laser light at a scattering angle of $90^{\circ}$ at $25^{\circ} \mathrm{C}$. The liposome solution was diluted 100-fold with PBS before measurement. Three measurements were taken for each sample. Transmission electron microscopy (Hitachi HT-7700, Japan) was used to observe the morphology of the liposomes. The liposome solution was diluted 10-fold with water and a drop of the diluted solution was placed on a carbon film and negatively stained with $1 \%$ phosphotungstic acid. After drying in air, sample analyses were carried out on a TEM with an acceleration of $100 \mathrm{kV}$.

The encapsulation efficiency (EE) of PTX in liposomes was quantified using a HPLC system. Liposome solution was diluted 10-fold with methanol, and was then eluted on an Agilent TC-C18 column $(250 \times 4.6 \mathrm{~mm}$ id, $5 \mu \mathrm{m}$ particles $)$ at a temperature of $30{ }^{\circ} \mathrm{C}$. The mobile phase consisted of acetonitrile and water (60:40,v/v), which was delivered at a flow rate of $1.0 \mathrm{~mL} \mathrm{~min}{ }^{-1}$. The injection volume was $10 \mu \mathrm{L}$ and the PTX absorbance was measured at a wavelength of $227 \mathrm{~nm}$. The EE was calculated by the following equation: $\mathrm{EE}=W / W_{0} \times 100 \%$, where $W_{0}$ and $W$ represent the amounts of PTX in the liposomes before and after passing over the Sephadex G-50 column, respectively.

The release profile of PTX from PFV-Lip-PTX was investigated using a dialysis method. Briefly, a volume of $1.0 \mathrm{~mL}$ of purified liposome suspension was mixed with $3 \mathrm{~mL}$ of RPMI-1640 medium containing $10 \%$ FBS, and transferred into a dialysis bag (MWCO 12000-14000 Da) sealed at both ends. Then the bag was immersed in $30 \mathrm{~mL}$ of release medium $(1.0 \mathrm{M}$ sodium salicylate) at $37{ }^{\circ} \mathrm{C}$, followed by gentle shaking at $100 \mathrm{rpm}$. At predetermined time intervals $(2,4,6,8,12$ and $24 \mathrm{~h}$ ), a $1.0 \mathrm{~mL}$ aliquot of the release medium was collected and replaced with an equal volume of fresh medium. After the $24 \mathrm{~h}$ period of the release test, the released amount of PTX in each time point was measured via HPLC.

\subsection{In vitro cellular uptake studies}

2.5.1 Flow cytometry analysis. For cellular uptake analysis using flow cytometry, MCF-7 cells were seeded into 12-well plates at a density of $1 \times 10^{5}$ cells per well and were allowed to attach overnight until $70-80 \%$ confluence was reached. Then the medium was removed and the cells were washed twice with PBS, and incubated with free Cou6, Lip-Cou6 and PFV-LipCou6, diluted with serum-free medium at the final concentration of $100 \mathrm{ng} \mathrm{mL}{ }^{-1}$ for $1 \mathrm{~h}$ at $37{ }^{\circ} \mathrm{C}$. Then, the cells were detached by trypsinization, pelleted by centrifugation and 
rinsed three times with cold PBS ( $\mathrm{pH}=7.4$ ). Finally, the mean Cou6 fluorescence intensity inside the cells was measured using a FACScan flow cytometer (Becton Dickinson FACSCalibur, USA), with 10000 events collected, excitation at $488 \mathrm{~nm}$ and detection at $560 \mathrm{~nm}$. Each assay was performed in triplicate.

2.5.2 Laser confocal microscopy analysis. For the confocal microscopy study, MCF-7 cells were cultured on microscope slides in a 24 -well plate $\left(5 \times 10^{4}\right.$ cells per well $)$ for $24 \mathrm{~h}$ until total adhesion was achieved. Then the cells were treated with free Cou6, Lip-Cou6 and PFV-Lip-Cou6 at a Cou6 concentration of $100 \mathrm{ng} \mathrm{mL} \mathrm{m}^{-1}$. After $1 \mathrm{~h}$ incubation, the culture medium was removed and the cells on the microscope slides were washed three times with ice-cold PBS. Then the cells were fixed with $4 \%$ paraformaldehyde solution for $20 \mathrm{~min}$, followed by cell nuclei staining with Hoechst $33258\left(10 \mu \mathrm{g} \mathrm{mL}^{-1}\right)$ for another $20 \mathrm{~min}$ and washing with PBS three times. Finally, fluorescent images of the cells were captured and analyzed using a LSM710 laser confocal microscope (Zeiss, Germany).

2.5.3 HPLC analysis. For the HPLC study, MCF-7 cells were seeded at a density of $3 \times 10^{5}$ cells per well in 6-well plate and incubated at $37^{\circ} \mathrm{C}$ for $24 \mathrm{~h}$ to allow cell attachment. Then the medium was removed and the cells were washed twice with PBS, and incubated with free PTX, Lip-PTX and PFV-Lip-PTX diluted with serum-free medium to a PTX final concentration of $10 \mu \mathrm{g}$ $\mathrm{mL}^{-1}$ for $3 \mathrm{~h}$ at $37^{\circ} \mathrm{C}$. Then, the cells were detached by trypsinization, pelleted by centrifugation and rinsed three times with cold PBS ( $\mathrm{pH}=7.4)$. Cells were then treated with RadioImmunoprecipitation Assay (RIPA) lysis buffer for $30 \mathrm{~min}$, and methanol was added to extract PTX. The extraction solution was centrifuged at $12000 \mathrm{rpm}$ for $10 \mathrm{~min}$ and the supernatant was analyzed using HPLC to measure PTX uptake by MCF-7 cells. HPLC detection conditions for PTX were similar to those described in Section 2.4.

\subsection{In vitro cytotoxicity studies}

The cytotoxic effects of free PTX, Lip-PTX and PFV-Lip-PTX against MCF-7 cells were determined using the sulforhodamine B (SRB) colorimetric assay. Briefly, MCF-7 cells were cultured in 96-well plates at a density of 5000 cells per well. After incubation for $24 \mathrm{~h}$, cells were treated with a series of concentrations of free PTX, Lip-PTX and PFV-Lip-PTX (0.25-256 ng $\mathrm{mL}^{-1}$ ) diluted with complete RPMI-1640 medium for $48 \mathrm{~h}$ at $37{ }^{\circ} \mathrm{C}$. Then the cells were fixed with $10 \%$ cold trichloroacetic acid, followed by washing and drying in the air. Subsequently, the fixed cells were stained with $0.4 \%$ SRB for $30 \mathrm{~min}$, washed with $1 \%$ acetic acid and dissolved in $10 \mathrm{mM}$ Tris base solution. Finally, the absorption representing cell viability was measured using a plate reader (Thermo Scientific, Multiskan FC, USA) at $540 \mathrm{~nm}$. All data were calculated as the percentages of viable cells relative to the survival of the control group (cells treated with medium) and presented as the mean $\pm \mathrm{SD}(n=3)$. The $\mathrm{IC}_{50}$ values were calculated using SPSS software.

\subsection{In vivo distribution study of PFV-Lip-DiR by live imaging}

The MCF-7 tumor xenograft model was established on BALB/c nude mice by subcutaneous inoculation of MCF-7 cells $(2 \times$
$10^{6}$ ) in the right armpit. When the tumor volume reached 50$100 \mathrm{~mm}^{3}$, the MCF-7 tumor-bearing mice were randomly divided into three groups and received intravenous injections of $0.2 \mathrm{~mL}$ free DiR, Lip-DiR and PFV-Lip-DiR via the tail vein. The final concentration of DiR was $5 \mu \mathrm{g} \mathrm{mL}{ }^{-1}$. At 2, 4, 8, 12, 24, 36 and $48 \mathrm{~h}$ after administration, mice were anesthetized with $2 \%$ isoflurane and the distributions of different formulations were visualized using an In Vivo Imaging System (Carestream Health, USA). After that, the mice were sacrificed immediately by cervical dislocation. The tumors and organs were collected and ex vivo fluorescence images were obtained on the same system.

\subsection{In vivo antitumor efficacy and toxicity studies}

In vivo antitumor efficacy was assessed using the xenograft tumor model as established above. When the tumor volume reached approximately $100 \mathrm{~mm}^{3}$, the mice were randomly assigned into 4 groups and treated with $0.2 \mathrm{~mL}$ of physiological saline, Taxol, LipPTX or PFV-Lip-PTX via the tail vein at a dosage of $10 \mathrm{mg}$ PTX/kg body weight on every second day for a total of 5 doses. The mice were then monitored every other day for tumor volumes and body weights. Tumor volume was calculated as $V=\left(\right.$ length $\times$ width $\left.^{2}\right) /$ 2. At the end point of the experiment, the mice were sacrificed, and the tumors were excised and weighed to evaluate the in vivo anti-tumor efficacy. The major organs were excised to make H\&E staining sections, which were observed using an optical microscope for histological analyses to evaluate the toxicity of the PTX solution and liposomes.

\subsection{Statistical analysis}

All the quantitative data were presented as the mean $\pm \mathrm{SD}$, and Student's $t$ test or one-way analysis of variance was performed to evaluate significance among groups. A value of $P$ less than 0.05 or 0.01 was considered to be statistically significant or highly significant.

\section{Results and discussion}

\subsection{Conjugation of PFV peptide with NHS-PEG-DSPE}

The targeting copolymer of PFV-PEG-DSPE was synthesized by conjugating isoleucine residue of PFV to NHS-PEG $2000-\mathrm{DSPE}$. According to the HPLC chromatograms in Fig. 2B, the retention time of unreacted PFV was about $21.2 \mathrm{~min}$ and the conjugation efficiency was about $79.2 \%$ after 6 days. These results indicate that PFV had been conjugated to PEG-DSPE. The formation of PFV-PEG $_{2000}$-DSPE was further confirmed using MALDI-TOF mass spectrometry. As shown in the MALDI-TOF MS spectra (Fig. 2C), the molecular weight (MW) of the reaction product was approximately $3655 \mathrm{Da}$, which was in good agreement with the theoretical MW, confirming that the targeting copolymer of PFV-PEG-DSPE had been successfully prepared.

\subsection{Preparation and characterization of various drug-loaded liposomes}

Fig. 2A shows a schematic representation of the PFV-modified liposomes. The physicochemical properties of PTX, coumarin6 (Cou6) and 1,10-dioctadecyltetramethyl indotricarbocy-anine 

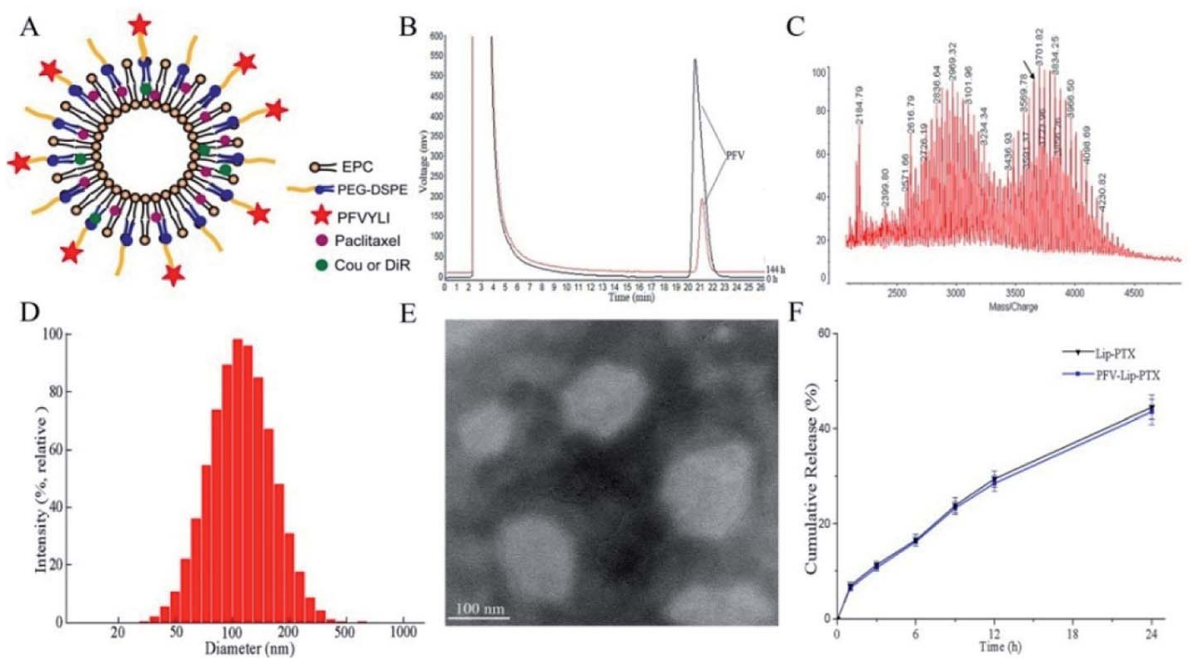

Fig. 2 Characteristics of PFV-modified liposomes loaded with PTX. (A) Schematic representation of PFV-modified Liposomes (PFV-Lip). (B) HPLC spectra monitoring the reaction of PFV with NHS-PEG $2000-D S P E$. (C) MALDI-TOF MS spectra of PFV-PEG $2000-D S P E$. (D) Size distribution graph of PFV-Lip-PTX obtained via dynamic light scattering. (E) Transmission electron microscopy (TEM) image of PFV-Lip-PTX. (F) In vitro release of PTX from Lip-PTX and PFV-Lip-PTX $(n=3)$.

iodide (DiR) loaded liposomes with or without PFV modification are summarized in Table 1 . Cou6 and DiR were used as hydrophobic fluorescent probes to investigate cellular uptake and the in vivo distribution of PFV-Lip. It has been previously reported that liposome size, especially aggregation size in the presence of serum, is one of the most important factors governing the fate of liposomes in vivo. ${ }^{30}$ In this study, the mean particle sizes of various liposomes were in the range of 105.4$123.0 \mathrm{~nm}$, with low polydispersity index (PDI) values of less than 0.25 . This size range is acknowledged as the best particle size for EPR. A typical particle size distribution graph of PFV-Lip-PTX is shown in Fig. 2D. The morphological structure was investigated using TEM (Fig. 2E). The TEM image shows that PFV-Lip-PTX comprises spheroid-like regular vesicles that are smaller than $200 \mathrm{~nm}$ in diameter, which is identical to the value obtained using DLS. Additionally, all liposomes are slightly charged with negative zeta potentials of less than $-3 \mathrm{mV}$, meaning that they are in the ideal charge range to accumulate in tumors efficiently and avoid nonspecific organ uptake. ${ }^{31}$ On the other hand, the EEs are all higher than $90 \%$. These results indicate that PFV modification would not bring about any significant changes to the physicochemical properties of the liposomes, and thus may not have any influence during the comparison of these two liposomal formulations. ${ }^{32}$
In vitro release studies are an essential step during drug development, because they not only allow for quality control of formulations, but also reveal the in vivo performance of the product. ${ }^{33}$ The release profiles of PTX from different liposomes are presented in Fig. 2F. No burst release was detected, and similar release kinetics was observed between Lip-PTX and PFVLip-PTX. The release rates of PTX in two liposomes were less than $10 \%$ at the initial $1 \mathrm{~h}$ and approximately $50 \%$ at $24 \mathrm{~h}$, indicating that the PTX remained encapsulated within the liposomes, thus leading to a delayed drug release. This is beneficial for tumor therapy, as it may prevent rapid leakage during systemic circulation and ensure that the PTX-loaded liposomes can reach and accumulate in the tumor sites.

Generally, the above results reveal that the PFV modification does not bring about visible changes to liposomes in terms of their size, surface potential, stability and release kinetics. This was favorable for the following comparison of these two liposomal systems in cell and animal studies.

\subsection{In vitro cellular uptake studies}

3.3.1 Flow cytometry analysis. To evaluate the ability of the PFV-modified liposomes to improve intracellular delivery, cellular uptake analysis was performed on MCF-7 cells by quantitative flow cytometry. Fig. 3A indicates that the cellular

Table 1 Characteristics of various drug-loaded liposomes $(n=3$, mean \pm SD)

\begin{tabular}{|c|c|c|c|c|}
\hline & Size $(\mathrm{nm})$ & PDI & Zeta potential & Encapsulation efficiency (\%) \\
\hline Lip-PTX & $119.0 \pm 5.3$ & $0.210 \pm 0.035$ & $-2.69 \pm 0.89$ & $92.2 \pm 4.3$ \\
\hline PFV-Lip-PTX & $123.0 \pm 7.1$ & $0.194 \pm 0.025$ & $-1.53 \pm 0.63$ & $90.7 \pm 2.6$ \\
\hline Lip-Cou6 & $110.8 \pm 5.7$ & $0.183 \pm 0.037$ & $-2.41 \pm 0.97$ & $93.3 \pm 1.9$ \\
\hline PFV-Lip-Cou6 & $106.8 \pm 4.5$ & $0.141 \pm 0.029$ & $-2.83 \pm 0.35$ & $95.6 \pm 3.1$ \\
\hline Lip-DiR & $105.4 \pm 4.8$ & $0.196 \pm 0.031$ & $-2.27 \pm 0.48$ & $94.1 \pm 2.5$ \\
\hline PFV-Lip-DiR & $109.4 \pm 3.2$ & $0.218 \pm 0.046$ & $-1.36 \pm 0.21$ & $92.1 \pm 3.2$ \\
\hline
\end{tabular}


A
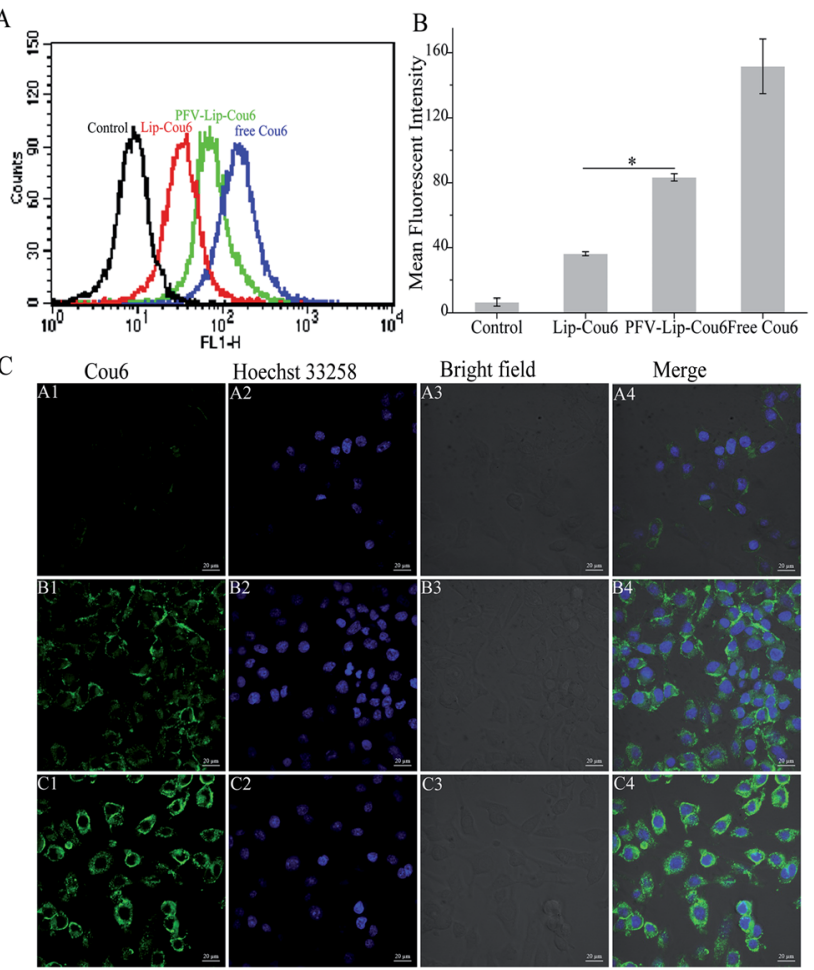

Fig. 3 Cellular uptake study of free Cou6, Lip-Cou6 and PFV-LipCou 6 on MCF- 7 cells at $37{ }^{\circ} \mathrm{C}$ for $1 \mathrm{~h}$. The final concentration of Cou6 was $100 \mathrm{ng} \mathrm{mL}^{-1}$. (A) Flow cytometric quantitative determination of Cou6 uptake. (B) Quantitative analysis of Cou6 uptake based on flow cytometric plots. Each bar represents the mean fluorescence intensity $\pm \mathrm{SD}(n=3), * p<0.01$. (C) Laser scanning confocal microscopy images of cells incubated with Lip-Cou6 (A1-4), PFV-Lip-Cou6 (B1-4) and free Cou6 (C1-4). Green and blue indicate the fluorescence of Cou6 and Hoechst 33258, respectively. Scale bars represent $20 \mu \mathrm{m}$

uptake of the PFV-modified liposomes was much higher than that of the unmodified liposomes. In more detail, the intracellular Cou6 level for PFV-Lip-Cou6 was 2.3-fold higher than that of Lip-Cou6 (Fig. 3B). The difference in cellular uptake between Lip-Cou6 and PFV-Lip-Cou6 actually indicates the difference between non-PFV and PFV modification, namely that PFV modification can facilitate the uptake of liposomes. Furthermore, the highly hydrophobic free Cou6, as a positive control, demonstrated the highest cellular Cou6 level, as it can go straight into the cell lipid membranes without release from liposomes, and then diffuse into the cell, leading to a greater extent of cellular accumulation. ${ }^{34}$

3.3.2 Laser confocal microscopy analysis. The intracellular accumulation and distribution of Cou6-loaded liposomes in MCF-7 cells was also analyzed using laser confocal microscopy. Fig. 3C shows the confocal microscopy images of MCF-7 cells after $1 \mathrm{~h}$ incubation with free Cou6, Lip-Cou6 and PFV-Lip-Cou6 at $37{ }^{\circ} \mathrm{C}$. Free Cou6, which was taken as the positive control group, showed the highest green fluorescence intensity, due to the same reason mentioned above. For PFV-Lip-Cou6, the microscopy images displayed more green fluorescence in the cell cytoplasm than Lip-Cou6, which demonstrated that PFV modification could enhance binding and penetration of the liposomes into the tumor cells. These results are in accordance with the above quantitative study of cellular uptake obtained from flow cytometry.

3.3.3 HPLC analysis. The cellular uptake of PTX in MCF-7 cells was also quantitatively evaluated using HPLC after different treatments with various PTX formulations. As is clearly shown in Fig. 4, the cellular uptake of the PTX formulations in the MCF-7 cells followed the order: free PTX > PFV-Lip-PTX > Lip-PTX. Compared to Lip-PTX, the PFV-Lip-PTX group showed 1.6 times higher intracellular PTX content, demonstrating a statistically significant difference. Free PTX as the positive control showed the highest intracellular PTX content due to the same reason mentioned above.

Ligand- or antibody-conjugated nanocarriers exhibit promising effects only in certain types of cancers that highly express the correlated specific receptors. ${ }^{35}$ However, patterns of receptor expression are diverse among different types of tumors and sometimes even within the same tumor, which limits the application of ligand- or antibody-based drug delivery systems. ${ }^{36}$ Theoretically, any interaction between cells and the specific molecules may be applied to enhance nanoparticle intracellular delivery. The hydrophobic interaction between hydrophobic peptides and cell membranes is another type of important interaction, which is often ignored or underestimated. ${ }^{21}$ The hydrophobic interaction is the primary driving force in a number of physiological events in the human body, including cell signaling, the formation of ion channels, cytolysis and cellular recognition. ${ }^{37}$ In this study, all the flow cytometry, confocal microscopy and HPLC results demonstrate that PFV modification obviously enhances the cellular uptake of liposomes on MCF-7 cells. This is attributed to the hydrophobic feature of PFV, which facilitates the interaction between the PFV-Lip and cell lipid membranes, thus improving the liposome intracellular uptake.

\subsection{In vitro cytotoxicity studies}

The first step in the therapeutic efficacy evaluation of the PFVLip-PTX formulation for breast cancer was to perform

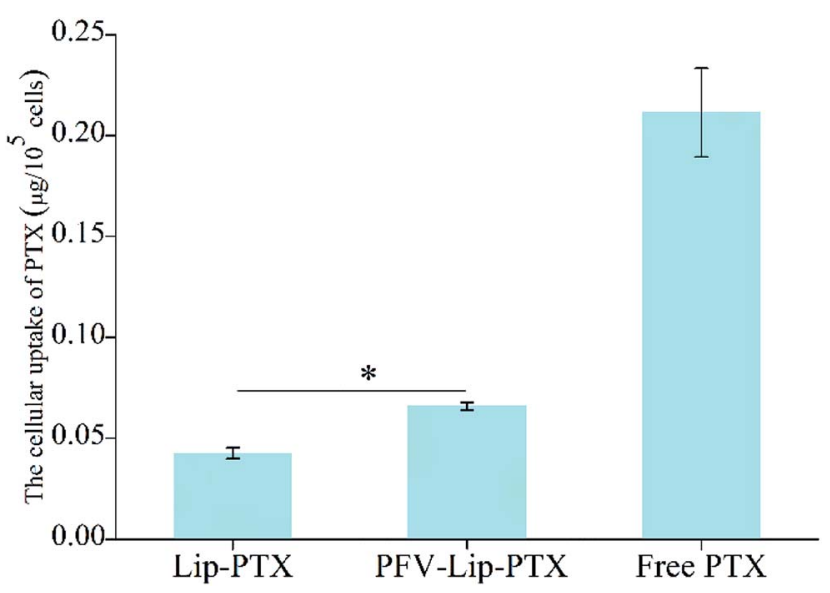

Fig. 4 Cellular uptake study of free PTX, Lip-PTX and PFV-Lip-PTX on MCF- 7 cells at $37^{\circ} \mathrm{C}$ for $3 \mathrm{~h}$. The final concentration of PTX was $10 \mu \mathrm{g}$ $\mathrm{mL}^{-1}$. Each bar represents mean $\pm \mathrm{SD}(n=3),{ }^{*} p<0.01$. 


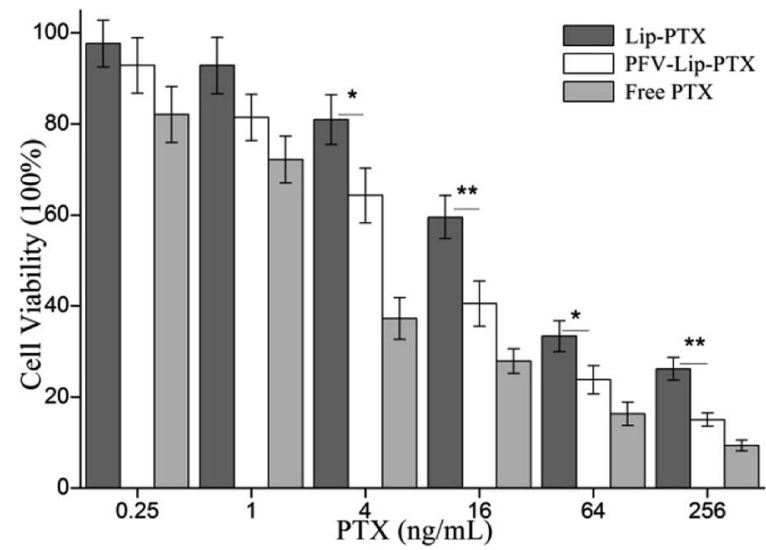

Fig. 5 In vitro cytotoxicity of free PTX, Lip-PTX, and PFV-Lip-PTX on MCF-7 cells by SRB assay after treatment with indicated concentrations of PTX formulations for $48 \mathrm{~h}$. Each bar represents mean $\pm \mathrm{SD}(n=$ 3). * and ** indicate statistically different $p<0.05$ and $p<0.01$.

a cytotoxicity assay on MCF-7 cells using an SRB method. Fig. 5 shows the cell viabilities of free PTX, Lip-PTX and PFV-Lip-PTX under a series of PTX concentrations against MCF-7 cells. The results demonstrate that free PTX, Lip-PTX and PFV-Lip-PTX could inhibit cell proliferation in a concentration-dependent manner. The $\mathrm{IC}_{50}$ values of free PTX, Lip-PTX and PFV-LipPTX were calculated to be $3.44 \pm 0.87,34.98 \pm 6.14$ and 11.23 $\pm 3.69 \mathrm{ng} \mathrm{mL} \mathrm{m}^{-1}$, respectively, indicating that the cytotoxicity of PFV-Lip-PTX was significantly higher than that of Lip-PTX. It is obvious that free PTX exhibited stronger cytotoxicity than the two liposomal PTX formulations. As expected, PFV modification significantly enhanced the cytotoxicity of liposomal PTX at a concentration range of $4-256 \mathrm{ng} \mathrm{mL}^{-1}$.

PTX could inhibit cell microtubule disassembly and promote the formation of unusually stable microtubules, thereby disrupting the normal dynamic reorganization of the microtubule network required for mitosis and cell proliferation, and finally causing cell apoptosis. ${ }^{38}$ Thus, in this study, we observed that PTX could inhibit MCF-7 cell proliferation in a concentration- dependent manner. The cytotoxicity of PTX formulations against the MCF-7 cells followed the order: free PTX > PFV-LipPTX > Lip-PTX. Free PTX exhibited better cytotoxicity with a lower $\mathrm{IC}_{50}$ value than Lip-PTX and PFV-Lip-PTX, owing to the different internalization mechanisms of free PTX and PTXloaded liposomes, and the sustained release manner of PTX from the liposomes. Free PTX could directly enter cells via passive diffusion and be effective, whereas the drug-loaded liposomes were mainly taken up by cells via the endocytic pathway and then the drug must be released to exert antitumor activity. ${ }^{39}$ The $\mathrm{IC}_{50}$ of the PFV-Lip-PTX group was found to be 3.1 times higher than that of Lip-PTX. The hydrophobic interaction of PFV with cell membranes could facilitate the cellular uptake of the liposomes, and then release PTX for anticancer effect. These results further confirm that the cellular uptake of the liposomes could be enhanced by the attachment of PFV on the surface of the liposomes, hence promoting the effectiveness of the PTX treatment.

\subsection{In vivo distribution study of PFV-Lip-DiR by live imaging}

To evaluate the in vivo biodistribution and tumor-targeting ability of the PFV-modified liposomes, free DiR, Lip-DiR and PFV-Lip-DiR were injected intravenously into mice bearing MCF-7 tumor xenografts and were monitored using a fluorescence imaging system. In the free DiR group, the fluorescence intensity in the tumor was negligible over a period of $48 \mathrm{~h}$, revealing no specific accumulation of free DiR in the tumor (data not shown). As shown in Fig. 6A, the fluorescence signals in the tumors were detected in both the Lip-DiR and PFV-LipDiR groups at $2 \mathrm{~h}$ after administration. Both groups displayed specific fluorescence distribution in tumors over time through passive targeting. However, the PFV-Lip-DiR group had high tumor targeting capability and resulted in stronger fluorescence intensity in the tumor than the Lip-DiR control group, especially from $12 \mathrm{~h}$ to $48 \mathrm{~h}$ after administration. More importantly, the tumor accumulation of DiR in the PFV-Lip-DiR group occurred much earlier and lasted longer at a higher level than that in Lip-
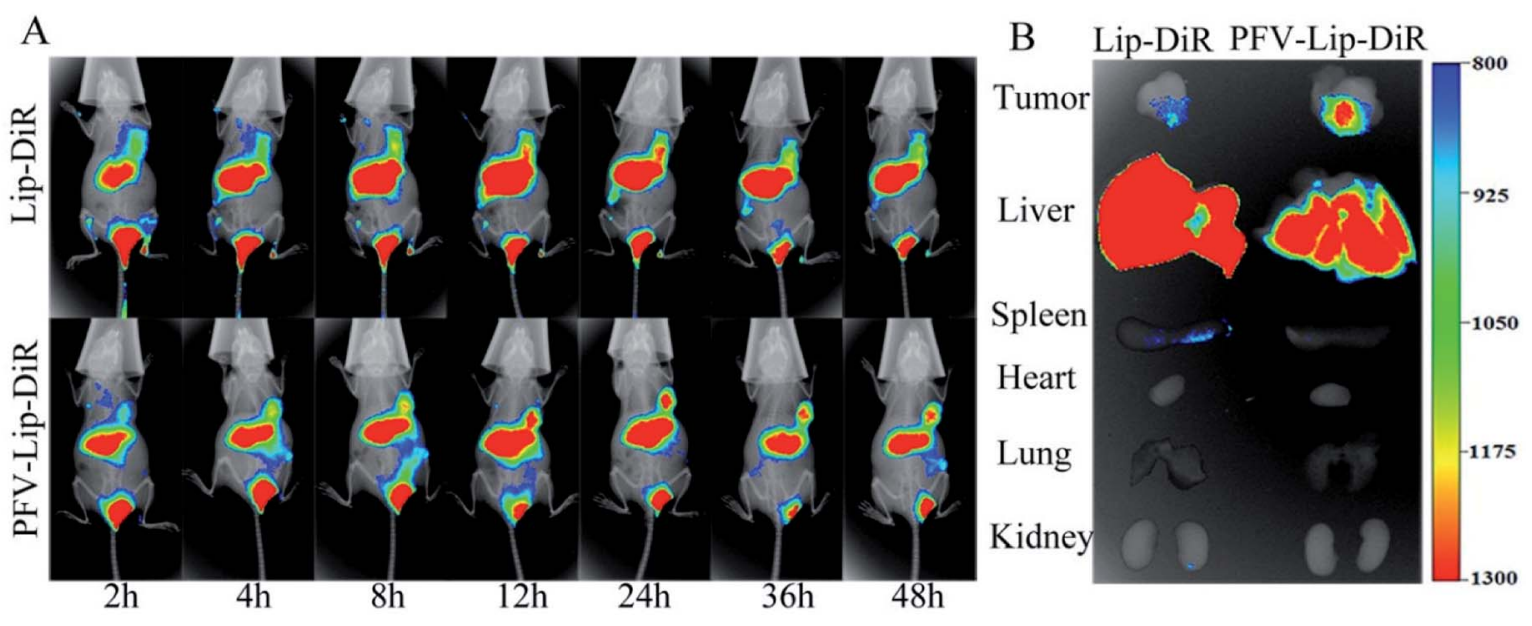

Fig. 6 (A) Representative whole-body in vivo fluorescence images of MCF-7 tumor-bearing BALB/c nude mice were injected intravenously with different DiR-loaded liposomes at different time points. (B) Ex vivo fluorescent images of dissected tumors and major organs of mice sacrificed after $48 \mathrm{~h}$ circulation in the body. 
DiR group. The fluorescence intensity began to decrease from $24 \mathrm{~h}$ post-injection in the Lip-DiR group, whereas in the PFVLip-DiR group, the fluorescence intensity in the tumors gradually increased and was still obvious until $48 \mathrm{~h}$. The ex vivo fluorescence images of the tumors and major organs at $48 \mathrm{~h}$ following i.v. injection revealed much stronger tumor fluorescence intensity in the PFV-Lip-DiR group than in the Lip-DiR group (Fig. 6B). As the typical tissue in RES, the livers and spleens had high fluorescence signals in both the Lip-DiR and Lip-SSLs-DiR groups, as expected. In brief, it was evident that the liposomes could target MCF-7 tumor tissue on the basis of the EPR effect after they were injected via the tail vein, and the PFV modification could further enhance the favorable distribution of PFV-Lip-DiR in the tumor site.

DiR, a near-infrared fluorescence probe, was utilized to label the liposomes as it could effectively reduce the interference caused by the animal auto-fluorescence. ${ }^{\mathbf{4 0}}$ Compared with unmodified Lip-DiR, PFV-Lip-DiR showed a stronger fluorescence distribution in the tumor from $12 \mathrm{~h}$ to $48 \mathrm{~h}$ after administration, which was still significant at $48 \mathrm{~h}$. It has been widely recognized that timely internalization by tumor cells can reduce the liposomes from re-entering systemic circulation. ${ }^{20}$ The higher tumor distribution of PFV-Lip is probably due to PFV modification increasing the internalization, before PFV-Lip could re-enter systemic circulation, allowing PTX to exert its therapeutic effects inside the cells. ${ }^{41}$

\subsection{In vivo antitumor efficacy and toxicity studies}

The PFV-modified liposomes showed definite antitumor effects in vitro. Therefore, the in vivo anti-tumor efficiency and systemic toxicity of PFV-Lip-PTX were further investigated. Fig. 7 displays the anti-tumor effects of various PTX formulations against MCF7 tumor-bearing BALB/c nude mice. Based on the tumor volume curve in Fig. 7A, tumor growth in the physiological saline group was more rapid than that in all PTX treatment groups. When compared to free PTX and Lip-PTX groups, the PFV-Lip-PTX group showed outstanding tumor suppression activity during the total test process and was significantly different $(p<0.05)$. At the end of the experiment, the ex vivo tumor weight of different treatment groups in Fig. 7B further confirmed that PFV-Lip-PTX exhibited significant tumor inhibition compared with Lip-PTX in the MCF-7 tumor-bearing mouse model. These results clearly indicate the greater tumor suppressive efficacy of our established PFV-Lip-PTX. The enhanced antitumor efficacy of PFV-Lip-PTX may be explained as follows: (1) negatively-charged PFV-Lip-PTX in circulation interacts less with plasma proteins, so it can resist non-specific protein absorption and escape RES rapid elimination; ${ }^{42}(2)$ the particle sizes of PFV-Lip-PTX are in the ideal range to take advantage of the EPR effect for tumor therapy, inducing greater accumulation of PFV-Lip-PTX into the tumor tissue via the EPR effect; ${ }^{43}$ (3) the liposomes possess excellent stability; and (4) PFV modification increases the cellular uptake of PFV-Lip-PTX to enhance cytotoxicity in breast cancer cells.

Mouse body weight changes in all groups, as an indicator of systemic toxicity, were measured simultaneously. As shown in Fig. 8, the body weights of mice in the control group slightly increased, and all the PTX treatment groups did not show sharp body weight loss. The body weight of mice in the Taxol group decreased to a greater extent than those in the PFV-Lip-PTXtreated group, suggesting the low systemic toxicity of PFV-LipPTX. The histological examination of several healthy organs of mice following treatment was conducted using H\&E staining (Fig. 9). H\&E staining showed that PFV-Lip-PTX did not cause visible histological changes to the major healthy organs over the duration of the experiment. These results confirm that PFVmodified liposomes can provide better anti-tumor effects with low systemic toxicity.

In addition to the treatment for breast cancer indicated in this study, we believe that the PFV-modified liposomes could be used for the delivery of different therapeutic or imaging agents to heterogeneous tumors, based on our studies into the mechanism of cellular uptake. Although the interaction between PFV and the tumor cell membrane is nonspecific, combined with the EPR effect of nano-sized liposomes, PFV-Lip shows potential to enhance the tumor tissue distribution and improve the antitumor therapy. In this way, this study with PFV is similar to
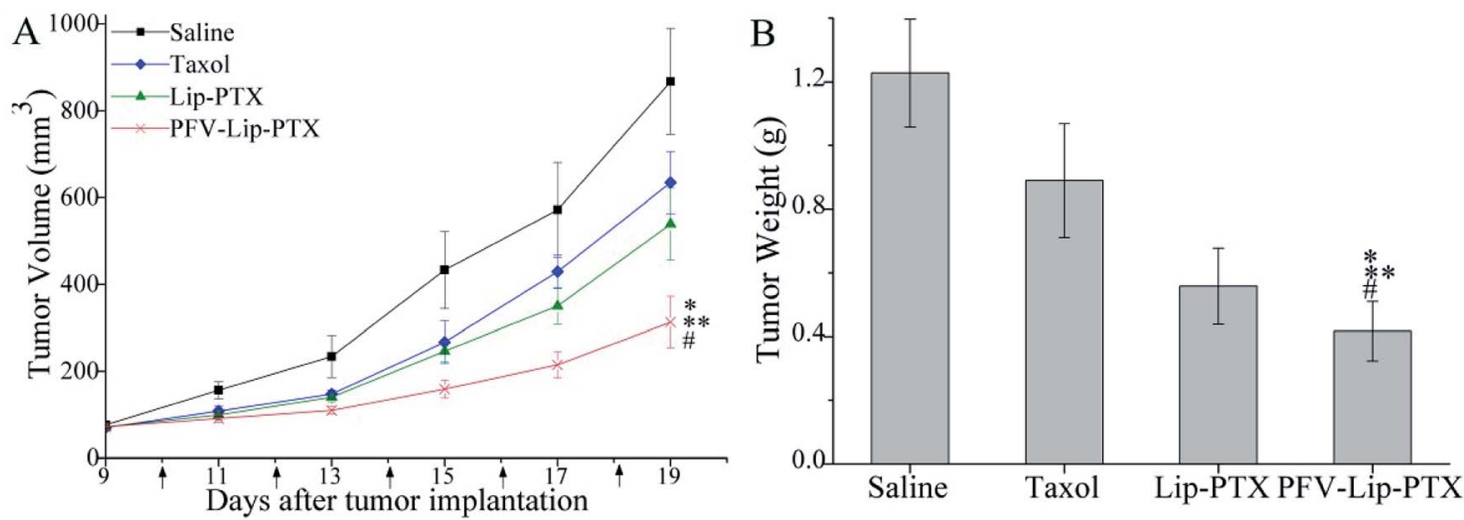

Fig. 7 In vivo antitumor efficacy and toxicity studies in MCF-7 human breast tumor-bearing BALB/c nude mice. Mice were treated with saline, Taxol, Lip-PTX and PFV-Lip-PTX every other day 5 times at a dosage of $10 \mathrm{mg} \mathrm{kg}^{-1}$ PTX. (A) Tumor volume changes of mice treated with different PTX-loaded formulations. (B) The weight of the excised tumor masses from different treatment groups. The black arrows note the day of administration. Data are presented as mean \pm SD $(n=6)$. Notes: ${ }^{*} p<0.01$ vs. saline; ${ }^{* *} p<0.05$ vs. taxol; ${ }^{\#} p<0.05$ vs. Lip-PTX. 


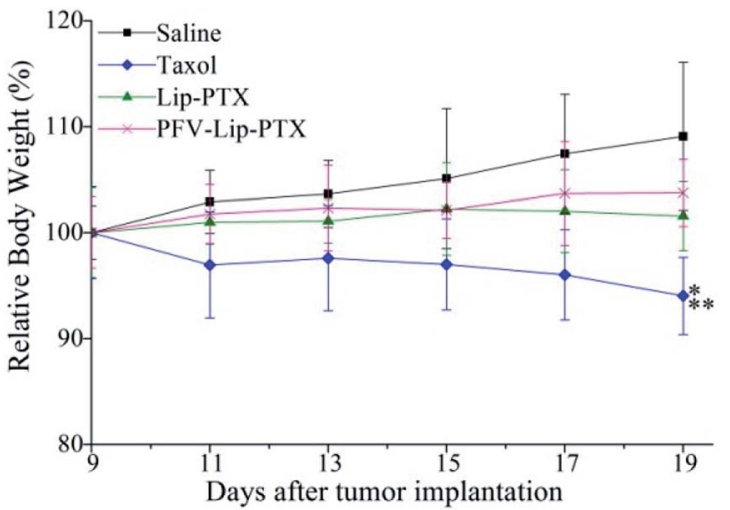

Fig. 8 Body weight changes of mice during anti-tumor efficacy study $(n=6)$. Notes: ${ }^{*} p<0.01$ vs. saline; ${ }^{*} p<0.05$ vs. PFV-Lip-PTX.

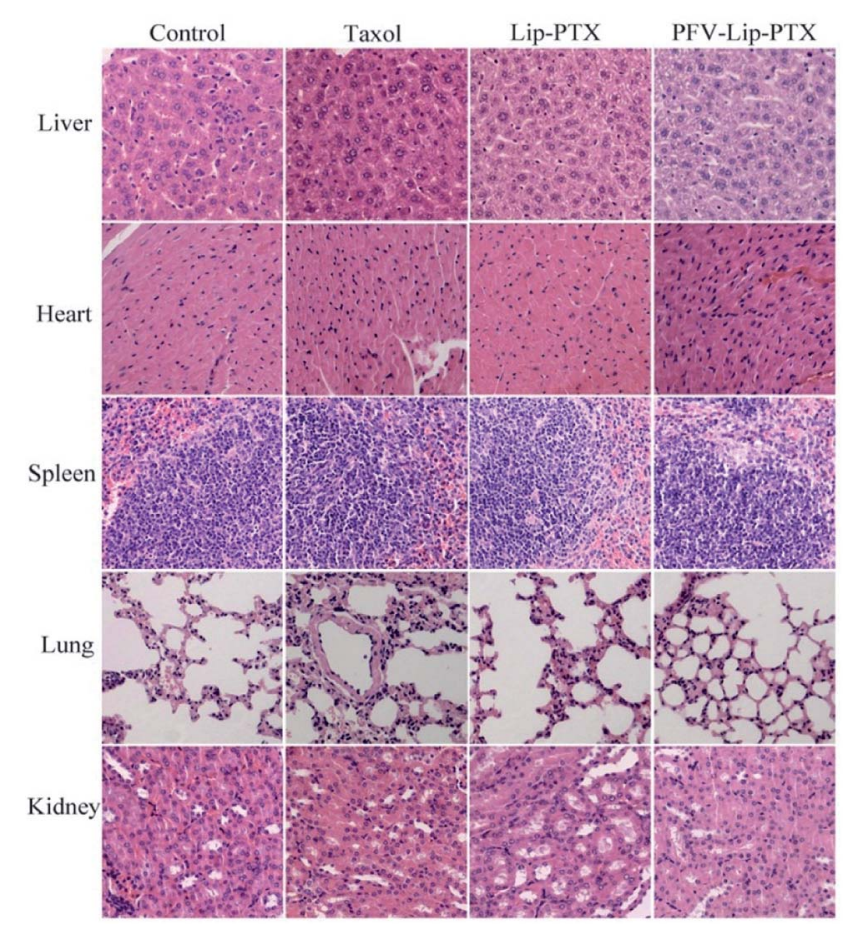

Fig. 9 Representative light microscopy images of several healthy organ $(\times 200)$ tissue sections with H\&E staining.

previous research on cyclosporine A and QLPVM-functionalized liposomes, and PFV-, CSA- and QLPVM-modified systems are generally similar to CPP- or target ligand-modified liposomes. ${ }^{20,21,44}$

\section{Conclusions}

PFVYLI, a low toxicity hydrophobic CPP, was successfully used to modify liposomes (PFV-Lip) to deliver PTX for the first time. PFV-Lip-PTX showed remarkable characteristics including ideal size and charge, narrow size distribution, high encapsulation efficiency and a controlled, sustained release profile. We demonstrated that PFV modification could enhance the binding of PFV-Lip to cancer cells via a hydrophobic interaction, and thus facilitate the intracellular delivery of liposomes into cancer cells. The results of cytotoxicity, in vivo distribution and antitumor efficacy experiments confirmed the superior therapeutic efficacy of PFV-Lip-PTX in vitro and in vivo. Furthermore, PFVLip-PTX displayed less systemic toxicity in terms of body weight changes and histological section observations. It is believed that PFV-Lip can provide us with a new perspective based on hydrophobicity, which is different to that exhibited by classic cationic CPPs. Consequently, PFV-Lip represents a promising platform for the improvement of therapeutic efficacy and reduction of drug toxicity, which may be widely applicable to the delivery of different types of chemotherapy drugs or imaging agents to various tumors.

\section{Conflicts of interest}

There are no conflicts to declare.

\section{Acknowledgements}

The project was financially supported by the Natural Science Foundation of Heilongjiang Province of China (No. H2015069).

\section{References}

1 N. Harbeck and M. Gnant, Lancet, 2017, 389, 1134-1150.

2 L. A. Torre, F. Bray, R. L. Siegel, J. Ferlay, J. Lortet-Tieulent and A. Jemal, Ca-Cancer J. Clin., 2015, 65, 87-108.

3 N. S. El Saghir, A. Tfayli, H. A. Hatoum, Z. Nachef, P. Dinh and A. Awada, Crit. Rev. Oncol. Hematol., 2011, 80, 433-449.

4 F. Ravar, E. Saadat, M. Gholami, P. Dehghankelishadi, M. Mahdavi, S. Azami and F. A. Dorkoosh, J. Controlled Release, 2016, 229, 10-22.

5 Y. Y. Shen, Z. K. Pi, F. Yan, C. K. Yeh, X. J. Zeng, X. F. Diao, Y. X. Hu, S. P. Chen, X. Chen and H. R. Zheng, Int. J. Nanomed., 2017, 12, 5613-5629.

6 K. L. Crossin and D. H. Carney, Cell, 1981, 27, 341-350.

7 S. P. Akhlaghi, S. Saremi, S. N. Ostad, R. Dinarvand and F. Atyabi, J. Nanomed. Nanotechnol., 2010, 6, 689-697.

8 A. Safavy, Curr. Drug Delivery, 2008, 5, 42-54.

9 P. Ma, S. R. Benhabbour, L. Peng and R. J. Mumper, Cancer Lett., 2013, 334, 253-262.

10 L. B. Ling, Y. W. Du, M. Ismail, R. Y. He, Y. P. Hou, Z. L. Fu, Y. Zhang, C. Yao and X. S. Li, Int. J. Pharm., 2017, 526, 11-22. 11 R. B. Weiss, R. C. Donehower, P. H. Wiernik, T. Ohnuma, R. J. Gralla, D. L. Trump, J. R. Baker Jr, D. A. Van Echo, D. D. Von Hoff and B. Leyland-Jones, J. Clin. Oncol., 1990, 8, 1263-1268.

12 Z. P. Zhang, L. Mei and S. S. Feng, Expert Opin. Drug Delivery, 2013, 10, 325-340.

13 S. Singh and A. K. Dash, Crit. Rev. Ther. Drug Carrier Syst., 2009, 26, 333-372.

14 P. P. Deshpande, S. Biswas and V. P. Torchilin, Nanomedicine, 2013, 8, 1509-1528.

15 X. Xu, L. Wang, H. Q. Xu, X. E. Huang, Y. D. Qian and J. Xiang, Asian Pac. J. Cancer Prev., 2013, 14, 2591-2594.

16 O. C. Farokhzad and R. Langer, ACS Nano, 2009, 3, 16-20. 
17 J. W. Sun, L. Jiang, Y. Lin, E. M. Gerhard, X. H. Jiang, L. Li, J. Yang and Z. W. Gu, Int. J. Nanomed., 2017, 12, 1517-1537.

18 C. P. Cerrato, K. Kunnapuu and U. Langel, Expert Opin. Drug Delivery, 2017, 14, 245-255.

19 D. M. Copolovici, K. Langel, E. Eriste and U. Langel, ACS Nano, 2014, 8, 1972-1994.

20 X. Y. Wang, X. H. Chen, X. C. Yang, W. Gao, B. He, W. B. Dai, H. Zhang, X. Q. Wang, J. C. Wang, X. Zhang, Z. F. Dai and Q. Zhang, J. Nanomed. Nanotechnol., 2016, 12, 387-397.

21 W. Gao, X. C. Yang, Z. Q. Lin, S. Y. Gao, B. He, D. Mei, D. Wang, L. Yuan, H. Zhang, W. B. Dai, X. Q. Wang, J. C. Wang, X. Zhang and Q. Zhang, J. Biomed. Nanotechnol., 2016, 12, 1183-1198.

22 J. R. Marks, J. Placone, K. Hristova and W. C. Wimley, J. Am. Chem. Soc., 2011, 133, 8995-9004.

23 X. Y. Liu, S. Timmons, Y. Z. Lin and J. Hawiger, Proc. Natl. Acad. Sci. U. S. A., 1996, 93, 11819-11824.

24 F. Nakayama, T. Yasuda, S. Umeda, M. Asada, T. Imamura, V. Meineke and M. Akashi, J. Biol. Chem., 2011, 286, 25823-25834.

25 D. F. Cai, W. Gao, B. He, W. B. Dai, H. Zhang, X. Q. Wang, J. C. Wang, X. Zhang and Q. Zhang, Biomaterials, 2014, 35, 2283-2294.

26 C. L. Watkins, P. Brennan, C. Fegan, K. Takayama, I. Nakase, S. Futaki and A. T. Jones, J. Controlled Release, 2009, 140, 237-244.

27 J. W. Park, E. K. Bang, E. M. Jeon and B. H. Kim, Org. Biomol. Chem., 2012, 10, 96-102.

28 M. Rhee and P. Davis, J. Biol. Chem., 2006, 281, 1233-1240.

29 B. Du, S. P. Han, H. Y. Li, F. F. Zhao, X. J. Su, X. H. Cao and Z. Z. Zhang, Nanoscale, 2015, 7, 5411-5426.

30 N. Maeda, Y. Takeuchi, M. Takada, Y. Sadzuka, Y. Namba and N. Oku, J. Controlled Release, 2004, 100, 41-52.
31 C. B. He, Y. P. Hu, L. C. Yin, C. Tang and C. H. Yin, Biomaterials, 2010, 31, 3657-3666.

32 M. A. Theresa, W. K. C. Wilson, I. H. Jennifer and M. L. Kimberley, Anti-Cancer Agents Med. Chem., 2006, 6, 513-523.

33 M. L. Van Slooten, O. Boerman, K. Romoren, E. Kedar, D. J. Crommelin and G. Storm, Biochim. Biophys. Acta, 2001, 1530, 134-145.

34 N. Düzgüneş and S. Nir, Adv. Drug Delivery Rev., 1999, 40, 318.

35 M. Li, H. Deng, H. Peng and Q. Wang, J. Nanosci. Nanotechnol., 2014, 14, 415-432.

36 R. K. Thapa, H. T. Nguyen, M. Gautam, A. Shrestha, E. S. Lee, S. K. Ku, H. G. Choi, C. S. Yong and J. O. Kim, Drug Delivery, 2017, 24, 1690-1702.

37 S. Kolusheva, T. Shahal and R. Jelinek, Biochemistry, 2000, 39, 15851-15859.

38 Y. Wang, S. Ma, Z. Xie and H. Zhang, Colloids Surf., B, 2014, 116, 41-48.

39 D. Zhu, S. Wu, C. Hu, Z. Chen, H. Wang, F. Fan, Y. Qin, C. Wang, H. Sun, X. Leng, D. Kong and L. Zhang, Acta Biomater., 2017, 58, 399-412.

40 P. Sharma, S. Brown, G. Walter, S. Santra and B. Moudgil, Adv. Colloid Interface Sci., 2006, 123, 471-485.

41 T. Lammers, F. Kiessling, W. E. Hennink and G. Storm, J. Controlled Release, 2012, 161, 175-187.

42 L. Jiang, L. Li, X. D. He, Q. Y. Yi, B. He, J. Cao, W. S. Pan and Z. W. Gu, Biomaterials, 2015, 52, 126-139.

43 E. Gullotti and Y. Yeo, Mol. Pharm., 2009, 6, 1041-1051.

44 K. Saar, M. Lindgren, M. Hansen, E. Eiríksdóttir, Y. Jiang, K. Rosenthal-Aizman, M. Sassian and Ü. Langel, Anal. Biochem., 2005, 345, 55-65. 